\title{
Relationship between area mortgage foreclosures, homeownership, and cardiovascular disease risk factors: The Hispanic Community Health Study/Study of Latinos
}

\author{
Earle C. Chambers ${ }^{1,2^{*}}$, David B. Hanna ${ }^{2}$, Simin Hua ${ }^{2}$, Dustin T. Duncan ${ }^{3}$, Marlene Camacho-Rivera ${ }^{4}$, \\ Shannon N. Zenk ${ }^{5}$, Jessica L. McCurley ${ }^{6}$, Krista Perreira ${ }^{7}$, Marc D. Gellman ${ }^{8}$ and Linda C. Gallo ${ }^{9}$
}

\begin{abstract}
Background: The risk of mortgage foreclosure disproportionately burdens Hispanic/Latino populations perpetuating racial disparities in health. In this study, we examined the relationship between area-level mortgage foreclosure risk, homeownership, and the prevalence of cardiovascular disease risk factors among participants of the Hispanic Community Health Study/Study of Latinos (HCHS/SOL).

Methods: HCHS/SOL participants were age 18-74 years when recruited from four U.S. metropolitan areas. Mortgage foreclosure risk was obtained from the U.S. Department of Housing and Urban Development. Homeownership, sociodemographic factors, and cardiovascular disease risk factors were measured at baseline interview between 2008 and 2011. There were 13,856 individuals contributing to the analysis (median age 39 years old, 53\% female).

Results: Renters in high foreclosure risk areas had a higher prevalence of hypertension and hypercholesterolemia but no association with smoking status compared to renters in low foreclosure risk areas. Renters were more likely to smoke cigarettes than homeowners.
\end{abstract}

Conclusion: Among US Hispanic/Latinos in urban cities, area foreclosure and homeownership have implications for risk of cardiovascular disease.

Keywords: Cardiovascular disease, Housing, Foreclosure, Homeownership

\section{Background}

Racial residential segregation made Hispanic/Latino and black households particularly vulnerable to predatory lending practices and these populations were thus hardest hit by the U.S. housing crisis beginning in 2007 [1]. Whether the housing crisis placed an additional burden on cardiovascular health in the Hispanic/Latino population is unclear. Hypertension, high cholesterol, and

\footnotetext{
* Correspondence: earle.chambers@einstein.yu.edu

'Department of Family \& Social Medicine, Albert Einstein College of

Medicine, 1300 Morris Park Avenue, Harold and Muriel Block Building, Rm.

409, Bronx, NY 10461, USA

${ }^{2}$ Department of Epidemiology \& Population Health, Albert Einstein College of Medicine, Bronx, NY 10461, USA

Full list of author information is available at the end of the article
}

smoking are referred to by the Centers for Disease Control and Prevention (CDC) as key risk factors for heart disease [2]. Nearly half of U.S. adults have at least one of these heart disease risk factors [3]. Prior studies examining the relationship between foreclosure and cardiovascular disease risk have included body mass index (BMI) [4-6], blood pressure [7-9], fasting glucose [7], mental health [10], and risk for hospitalization for heart attack and stroke $[9,11,12]$. These results, however, have been inconsistent. For example, Arcaya and colleagues [8] showed that adults living in close proximity to a foreclosed property were more likely to have elevated blood pressure, whereas Christine et al. [7] showed a small inverse relationship between foreclosures in a neighborhood

(c) The Author(s). 2019 Open Access This article is distributed under the terms of the Creative Commons Attribution 4.0 International License (http://creativecommons.org/licenses/by/4.0/), which permits unrestricted use, distribution, and reproduction in any medium, provided you give appropriate credit to the original author(s) and the source, provide a link to the Creative Commons license, and indicate if changes were made. The Creative Commons Public Domain Dedication waiver (http://creativecommons.org/publicdomain/zero/1.0/) applies to the data made available in this article, unless otherwise stated. 
and blood pressure. Studies of associations of area foreclosures and BMI are similarly inconsistent [4-6]. Inconsistent findings among these studies may reflect differences in measurement of foreclosures at the neighborhood levels or possibly differing mechanisms driving the association between foreclosures and various cardiovascular disease risk factors.

As research further uncovers the mechanisms linking foreclosures to health, examining the role of homeownership can be an important step in guiding policy. Homeowners are generally considered the most vulnerable during a foreclosure crisis but studies showing an association between neighborhood foreclosures and health suggest that both homeowners and renters can be affected by high foreclosures in the area. [4, 11, 12]. A high level of neighborhood foreclosures may influence health outcomes by reflecting conditions that contribute to environmental-related stress of residents $[8,10]$; limited access to health care [11, 12]; and/or limited access to resources for a healthy diet and physical activity for residents [13]. More research is needed to confirm prior findings of the association of neighborhood foreclosures and cardiovascular disease. These studies should characterize the risk among homeowners and renters and examine a wider range of cardiovascular disease risk factors such as high cholesterol and cigarette smoking.

Despite a growing literature on the associations between neighborhood foreclosure and health [14], as well as homeownership and health [15], existing studies examining cardiovascular disease risk factors suffer from a variety of limitations. First, most studies do not examine more nuanced associations between neighborhood foreclosure, homeownership, and health. For example, among residents living in proximity to foreclosed properties, it is not clear whether residents who rent are as likely to show the poor health-related association of living in a high foreclosure risk neighborhood as residents who own their own home. Second, few studies include health behaviors associated with cardiovascular disease such as smoking. Third, many studies have not included large samples of racial and ethnic minorities, including Hispanic/Latinos - the largest ethnic minority population in the U.S. Fourth, many studies focus on single cities or limited geographical areas, which limits generalizability. The purpose of this study was to examine the relationship between neighborhood foreclosure risk, homeownership, and cardiovascular disease risk factors - i.e., hypertension, hypercholesterolemia, cigarette smoking - among Hispanic/Latino adults living in 4 major metropolitan areas in the U.S. We further examined whether the association of neighborhood foreclosure risk on cardiovascular disease risk factors differed between homeowners and renters, rarely addressed in previous research.

\section{Methods}

\section{Study population and design}

The Hispanic Community Health Study/Study of Latinos (HCHS/SOL) is a community-based prospective cohort study of 16,415 self-identified Hispanic/Latino persons aged 18-74 years at screening from randomly selected households in four U.S. field centers (Chicago, IL; Miami, FL; Bronx, NY; San Diego, CA) with baseline examination (2008 to 2011) and yearly telephone follow-up assessment. The goals of the HCHS/SOL, sample design, and cohort selection have been previously described $[16,17]$. The baseline clinical examination included comprehensive biological (e.g., anthropometrics, blood draw), behavioral (e.g., tobacco use assessed by self-report), and socio-demographic (e.g., socioeconomic status, nativity) assessments. The Institutional Review Board at each field center approved the study. All participants gave written informed consent in either English or Spanish.

\section{Exposures of interest Mortgage foreclosure risk}

In 2008, the U.S. Department of Housing and Urban Development (HUD) created a novel mortgage foreclosure risk metric which estimates mortgage foreclosure risk for the year 2007 and the first six months of 2008 as a function of area decline in home values as of June 2008; unemployment rate as of June 2008; and high cost mortgage loans between 2004 and 2006. The mortgage foreclosure risk metric is estimated at the census tract level and reflects areas in the country that have started or could potentially become areas of abandonment and disinvestment. This measure was used to inform where state and local resources should be targeted to stabilize neighborhoods and stem the decline of house values of homes in these areas. More details on the methodology HUD used to calculate mortgage foreclosure risk is available on the HUD website [18].

\section{Homeownership}

Homeownership was determined by a question asked during the baseline HCHS/SOL visit: Is your house, apartment, or mobile home... (1) "Owned by you or someone in the household free and clear --- without a mortgage or loan"; (2) "Owned by you or someone in the household--- with a mortgage or loan"; (3) "Rented"; or (4) occupied without rent. In order to be consistent with other studies that do not distinguish between mortgage status among owners, both of the 'owned' categories were combined into one category and compared with renters. $[19,20]$

\section{Cardiovascular disease risk factors}

Each cardiovascular disease risk factor was measured during the baseline clinic visit of $\mathrm{HCHS} / \mathrm{SOL}$ 
participants. Three seated blood pressure measurements were obtained after a 5-min rest period using an automatic sphygmanometer. The average of the second and third measurement was used in analysis. Hypertension was defined as a systolic blood pressure $\geq 140 \mathrm{mmHg}$, diastolic blood pressure $\geq 90 \mathrm{mmHg}$, and/or receiving antihypertensive medication. Hypercholesterolemia was defined as total cholesterol $\geq 240 \mathrm{mg} / \mathrm{gL}$, LDL cholesterol $\geq 160 \mathrm{mg} / \mathrm{dL}$, or HDL cholesterol $<40 \mathrm{mg} / \mathrm{dL}$ or receiving cholesterol lowering medications. Cigarette smoking was categorized as never, former, and current use.

\section{Covariates}

Participants' height was measured to the nearest centimeter and body weight to the nearest $0.1 \mathrm{~kg}$. BMI was calculated as weight in kilograms divided by height in meters squared. BMI categories were defined as underweight $\left(<18.5 \mathrm{~kg} / \mathrm{m}^{2}\right)$, normal weight $\left(18.5-24.9 \mathrm{~kg} / \mathrm{m}^{2}\right)$, overweight $\left(25.0-29.9 \mathrm{~kg} / \mathrm{m}^{2}\right)$, and obese $\left(\geq 30.0 \mathrm{~kg} / \mathrm{m}^{2}\right)$. Potential confounders. Socio-demographic characteristics self-reported during the baseline exam included: age, sex, household income, education, employment, nativity (foreign-born vs. US-born), and Hispanic/Latino background. Neighborhood percent poverty was defined as the percentage of families per census tract (CT) whose income in the past 12 months was below the poverty line, based on data from 2007 to 2011 American Community Survey 5 -year estimates [21, 22].

\section{Statistical analyses}

All participants of the HCHS/SOL cohort with complete information for study variables were included in the current analysis $(n=13,856)$ with several specific exceptions. Residents indicating that they occupy their home without paying rent were excluded from analysis $(n=$ 422). Since we were interested in examining risk factor for cardiovascular disease and to reduce the likelihood of reverse causation of residents with pre-existing cardiovascular disease preferentially residing in high foreclosure areas, participants with preexisting cardiovascular disease at the baseline interview were also excluded from analysis $(n=1166)$. Preexisting cardiovascular disease included prevalent coronary heart disease, defined as self-report of history of heart attack or procedure (angioplasty, stent, bypass) or electrocardiogram showing old myocardial infarction; or cerebrovascular disease, defined as self-reported medical history of stroke, mini-stroke or transient ischemic attack, or carotid revascularization or balloon angioplasty or surgery to the arteries in the neck at baseline assessment.

A mortgage foreclosure risk value was attributed to each participant based on his or her residential census tract. The mortgage foreclosure risk variable was linked based on 2000 census tract boundaries, whereas the percent poverty variable was linked to each participant's census tract based on 2010 census tract boundaries. In this study, 97\% of addresses were successfully geocoded. Participants not able to be geocoded were dropped from the analysis $(n=551)$.

We computed descriptive statistics (e.g. proportions) across all study variables. We initially compared all study variables including homeownership across tertiles of mortgage foreclosure risk. Poisson regression models were used to estimate prevalence ratios (PR) with 95\% confidence intervals $(95 \% \mathrm{CI})$ for hypertension, hypercholesterolemia, and smoking by homeownership status and mortgage foreclosure risk, with robust variance estimation used to account for clustering by census tract. Additional stratified analyses were done to examine the association of mortgage foreclosure risk with cardiovascular disease risk factors by homeownership status. Analyses were primarily adjusted for age, sex, education level, employment status, income level, nativity, Hispanic/Latino group, and percent poverty level.

All reported values (means, prevalences, and prevalence ratios) were weighted to account for the disproportionate selection of the sample and to partially adjust for any bias due to differential nonresponse in the selected sample at the household and individual levels. The adjusted weights were also trimmed to limit precision losses due to the variability of the adjusted weights, and calibrated to the 2010 Census characteristics by age, sex, and Hispanic/Latino background in each field site's target population. All analyses also account for cluster sampling and the use of stratification in sample selection.

Statistical significance was determined at the $P<$ 0.05 level. All analyses were performed using SAS 9.4 software (SAS Institute, Cary, NC) and SUDAAN software Release 11.0 (RTI International, Research Triangle Park, NC).

\section{Results}

There were 13,856 HCHS/SOL participants whose data contributed to the study. Table 1 shows the distribution of baseline characteristics of HCHS/SOL participants by tertile of neighborhood mortgage foreclosure risk. Most HCHS/SOL participants lived in a rental unit (74\%). A higher percentage of renters lived in high mortgage foreclosure risk areas, than low mortgage foreclosure risk areas; and more homeowners lived in low mortgage foreclosure risk areas than in high mortgage foreclosure risk areas. Participants who identified as Cuban were largely located in areas of high foreclosure risk, while participants who identified as Mexican were largely in areas of low and medium foreclosure risk (Table 1). The census tract-level correlation between mortgage foreclosure risk and percent poverty was $r=0.16(P<0.01)$, 
Table 1 Demographic, homeownership status, and health characteristics of the HCHS/ SOL cohort by census tract-level mortgage foreclosure risk

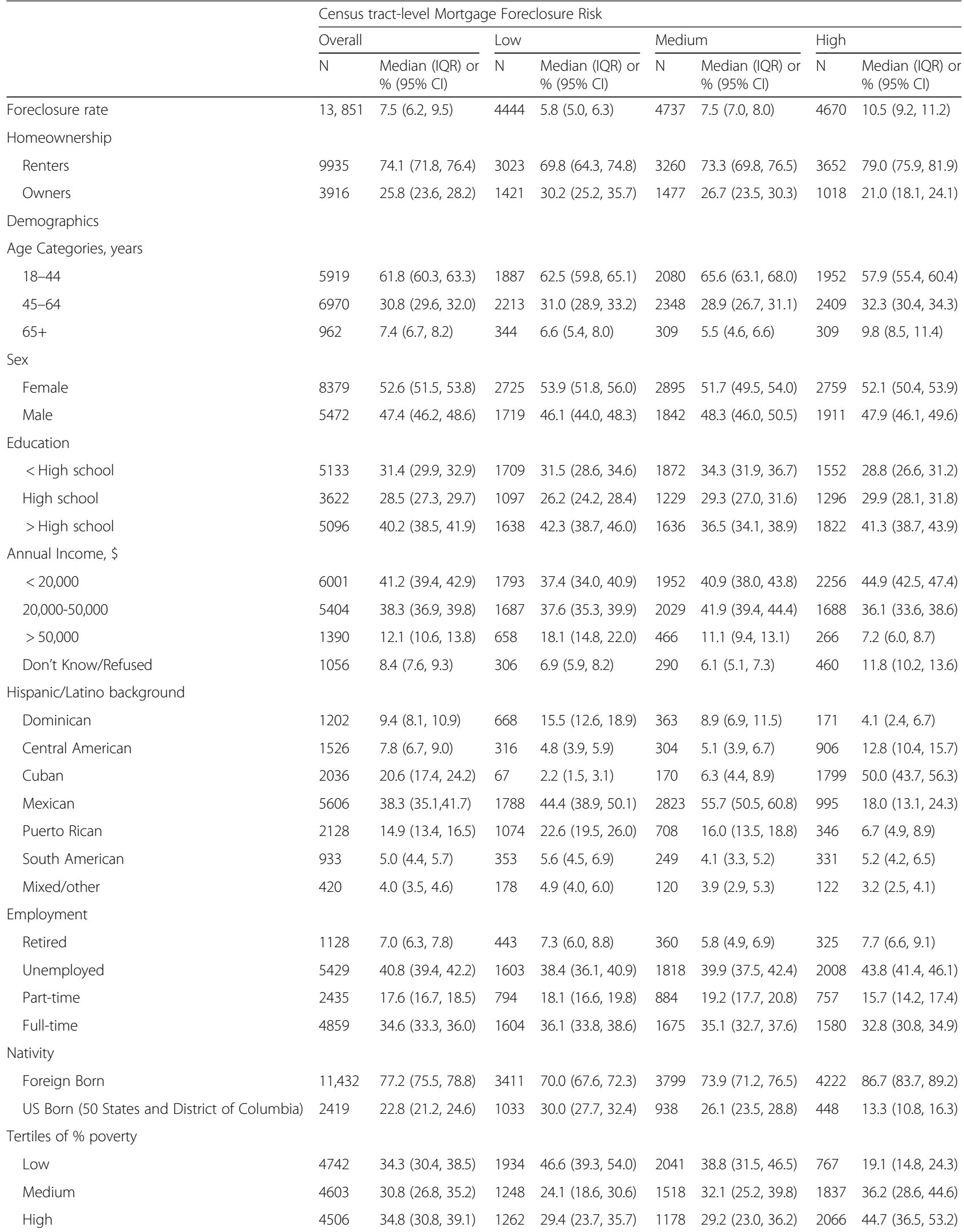


Table 1 Demographic, homeownership status, and health characteristics of the HCHS/ SOL cohort by census tract-level mortgage foreclosure risk (Continued)

\begin{tabular}{|c|c|c|c|c|c|c|c|c|}
\hline & \multicolumn{8}{|c|}{ Census tract-level Mortgage Foreclosure Risk } \\
\hline & \multicolumn{2}{|c|}{ Overall } & \multicolumn{2}{|l|}{ Low } & \multicolumn{2}{|c|}{ Medium } & \multicolumn{2}{|l|}{ High } \\
\hline & $\mathrm{N}$ & $\begin{array}{l}\text { Median (IQR) or } \\
\%(95 \% \mathrm{Cl})\end{array}$ & $\mathrm{N}$ & $\begin{array}{l}\text { Median (IQR) or } \\
\%(95 \% \mathrm{Cl})\end{array}$ & $\mathrm{N}$ & $\begin{array}{l}\text { Median (IQR) or } \\
\%(95 \% \text { CI) }\end{array}$ & $\mathrm{N}$ & $\begin{array}{l}\text { Median (IQR) or } \\
\%(95 \% \mathrm{Cl})\end{array}$ \\
\hline \multicolumn{9}{|l|}{ Health } \\
\hline Hypertension & 3806 & $21.8(20.7,23.1)$ & 1243 & $19.4(17.6,21.3)$ & 1173 & $18.8(16.9,20.8)$ & 1390 & $26.7(24.7,28.9)$ \\
\hline Hypercholesterolemia & 6064 & $40.6(39.3,41.8)$ & 1903 & $38.1(35.9,40.3)$ & 2015 & $39.1(36.9,41.3)$ & 2146 & $44.2(42.2,46.2)$ \\
\hline Current Cigarette Use & 2616 & $20.7(19.6,21.8)$ & 753 & $18.4(16.7,20.1)$ & 833 & $19.9(18.1,21.9)$ & 1030 & $23.5(21.7,25.5)$ \\
\hline \multicolumn{9}{|l|}{$\mathrm{BMl}, \mathrm{kg} / \mathrm{m}^{2}$} \\
\hline Underweight $(<18.5)$ & 106 & $1.1(0.9,1.5)$ & 34 & $1.5(0.9,2.3)$ & 27 & $0.6(0.4,1.0)$ & 45 & $1.2(0.8,1.8)$ \\
\hline Normal (18.5-24.9) & 2750 & $22.7(21.7,23.8)$ & 878 & $22.7(21.0,24.5)$ & 911 & $21.8(19.9,23.8)$ & 961 & $23.5(21.9,25.1)$ \\
\hline Overweight (25-29.9) & 5244 & $37.6(36.3,38.8)$ & 1681 & $38.0(35.6,40.4)$ & 1810 & $37.9(35.6,40.3)$ & 1753 & $36.9(35.238 .6)$ \\
\hline Obese $(\geq 30)$ & 5751 & $38.6(37.3,39.9)$ & 1851 & $37.9(35.5,40.3)$ & 1989 & $39.6(37.3,42.1)$ & 1911 & $38.4(36.5,40.4)$ \\
\hline \multicolumn{9}{|l|}{ Center } \\
\hline Bronx & 3204 & $26.8(24.1,29.7)$ & 1900 & $46.1(39.7,52.7)$ & 987 & $27.5(21.8,34.0)$ & 317 & $8.0(4.9,12.8)$ \\
\hline Chicago & 3662 & $16.7(14.7,18.8)$ & 1472 & $21.0(16.5,26.3)$ & 1301 & $19.9(15.0,26.0)$ & 889 & $9.8(6.3,15.0)$ \\
\hline Miami & 3543 & $30.3(26.2,34.8)$ & 94 & $2.9(1.7,4.7)$ & 347 & $10.9(7.8,15.0)$ & 3102 & $72.4(64.8,79.0)$ \\
\hline San Diego & 3442 & $26.3(23.0,29.8)$ & 978 & $30.0(23.1,38.0)$ & 2102 & $41.7(35.1,48.6)$ & 362 & $9.8(5.7,16.2)$ \\
\hline
\end{tabular}

Census tract-level Mortgage Foreclosure Risk: Low: foreclosure rates $\leq 6.65 \%$; Medium: $6.65-8.44 \%$; High: $>8.44 \%$ in the HCHS/SOL cohort

Hypertension is defined as: systolic or diastolic BP is greater than or equal to $140 / 90 \mathrm{mmHg}$ or if the participant self-reported as currently taking antihypertensive mediations. Participants without a blood pressure measurement and no medication use were assumed to be not hypertensive

Dyslipidemia is defined as: total cholesterol $\geq 240$ or Low Density Lipoprotein cholesterol $\geq 160$ or High Density Lipoprotein cholesterol $<40$ or use of lipid lowering drugs

indicating that high mortgage foreclosure risk areas were not uniformly congruent with high poverty areas.

Crude PR showed that each increase in tertile of foreclosure was associated with an increasing trend in prevalence of hypertension and current smoking status and a decreasing trend in hypercholesterolemia [Table 2]. In adjusted models, high foreclosure risk was positively associated with prevalence of hypertension and hypercholesterolemia among renters but not among homeowners [Table 2]. Homeownership was associated with a lower

Table 2 Multivariable Poisson regression analysis of census tract-level mortgage foreclosure risk and prevalence of cardiovascular disease risk factors stratified by homeownership status among HCHS/SOL participants

\begin{tabular}{|c|c|c|c|c|}
\hline & \multicolumn{2}{|l|}{ Owners } & \multicolumn{2}{|l|}{ Renters } \\
\hline & Crude PR (95\% Cl) & Adjusted $^{\text {a }}$ PR $(95 \%$ Cl) & Crude PR (95\% Cl) & Adjusted $^{\mathrm{a}}$ PR $(95 \% \mathrm{Cl})$ \\
\hline \multicolumn{5}{|c|}{ Census tract-level mortgage foreclosure risk } \\
\hline \multicolumn{5}{|l|}{ Hypertension } \\
\hline High & $1.09(0.87,1.37)$ & $0.87(0.68,1.11)$ & $1.52(1.31,1.76)$ & $1.25(1.08,1.46)$ \\
\hline Medium & $0.90(0.71,1.14)$ & $0.99(0.84,1.17)$ & $1.01(0.86,1.20)$ & $1.10(0.96,1.28)$ \\
\hline Low (referent) & - & - & - & - \\
\hline \multicolumn{5}{|c|}{ Hypercholesterolemia } \\
\hline High & $0.89(0.75,1.05)$ & $0.94(0.80,1.11)$ & $1.04(0.95,1.14)$ & $1.12(1.01,1.24)$ \\
\hline Medium & $0.98(0.85,1.14)$ & $0.98(0.84,1.13)$ & $1.14(1.03,1.25)$ & $1.01(0.92,1.11)$ \\
\hline Low (referent) & - & - & - & - \\
\hline \multicolumn{5}{|l|}{ Current Smoking } \\
\hline High & $1.27(0.97,1.67)$ & $1.16(0.80,1.68)$ & $1.10(0.95,1.27)$ & $1.12(0.95,1.31)$ \\
\hline Medium & $1.22(0.91,1.63)$ & $0.95(0.72,1.25)$ & $1.02(0.86,1.20)$ & $1.06(0.91,1.24)$ \\
\hline Low (referent) & - & - & - & - \\
\hline
\end{tabular}

Cl confidence interval, $P R$ prevalence ratio. Census tract-level mortgage foreclosure risk: Low: foreclosure rates $\leq 6.65 \%$; Medium: $6.65-8.44 \%$; High: $>8.44 \%$ in the $\mathrm{HCHS} / \mathrm{SOL}$ cohort. ${ }^{a}$ Adjusted for age, sex, education, income, ethnicity, body mass index, cigarette use (except in smoking model), nativity, employment, and neighborhood poverty 
prevalence of smoking (APR: 0.83; 95\% CI: 0.72-0.96) but was not associated with hypertension (APR: 1.08; 95\% CI: 0.98-1.18), or hypercholesterolemia (APR: 0.98; 95\% CI: 0.90-1.06) in adjusted models including foreclosure risk, BMI, and confounding variables.

\section{Discussion}

The HUD mortgage foreclosure risk metric identifies at-risk neighborhoods on the verge of economic instability and crisis. It is a measure that captures areas starting to decline providing a window to prevention where policy may influence cardiovascular disease. Using a sample of Hispanics/Latinos sheds light on the health consequences of a largely growing demographic in the U.S. hardest hit by the 2007 housing crisis. Our results showing a significant association of area foreclosure risk with hypertension and hypercholesterolemia among renters but not homeowners suggest that renters are particularly vulnerable in areas where the risk for foreclosures is high. The null finding among homeowners may suggest that residents owning their homes are better able to weather the economic downturn than renters. This is consistent with data showing that homeowners have stronger social ties within the communities that they live in and are better able to access the resources in their neighborhood that promote healthier lifestyle choices and overall wellbeing [23-25]. In our study, we also showed that renters were more likely to be current cigarette smokers than homeowners. This is consistent with reports showing smoking risk to be almost three times more likely among renters than homeowners [26]. Renting, in this study, may indicate a less stable housing environment; and living in less stable housing undermines prioritizing healthy behaviors [27, 28].

Saegert proposes a model where home foreclosures also contribute to racial disparities in housing and place racial and ethnic minorities in housing niches where once a household is in this niche they are exposed to cumulative hazards that affect current and future generations [29, 30]. This is an interesting approach to understanding the health consequences of area foreclosures as a contextual exposure influencing access to resources over time. More research is needed to further elucidate the pathways that might link neighborhood foreclosures and cardiometabolic disease. Considering the intersection of foreclosure, homeownership, and health can inform housing policies with the potential to broaden public health impact and reduce racial and ethnic and socio-economic status-related health disparities $[31,32]$.

The strength of our foreclosure metric is that it is consistent with federal guidelines used to determine the allocation of resources to communities in most need. The health consequences of the foreclosure crisis that preceded the Great Recession affected renters as well as homeowners. [9] In our study, we posit that neighborhood foreclosure risk is a useful measure of the neighborhood cost of economic decline particularly as it relates to housing insecurity, which can affect all of those living in an area, renters and homeowners alike. Foreclosure risk as measured in this study has some precedent in its use in understanding adverse health outcomes. In a sample of breast cancer survivors, Schootman et al. [20] used a HUD predicted measure of area foreclosures to show that women living in high foreclosure risk areas are more likely to report fair-poor health than women in low foreclosure risk areas. This suggests that residents can begin to feel the distress of living in an at-risk area prior to an actual increase in foreclosure rates. This measure is directly applicable to federal housing-related policies that use this metric to distribute aid to vulnerable communities.

\section{Limitations}

This study is cross-sectional; therefore, it is not possible to determine the temporal relationship between foreclosure, homeownership, and cardiovascular disease risk factors. By limiting our analysis to participants without cardiovascular disease, however, we sought to limit reverse causation where participants with illness were more likely to be in a neighborhood with more foreclosure risk and less likely to be a homeowner. Furthermore, all analyses assume no residential movement between mortgage foreclosure risk assessment and the baseline interview/clinical assessment of HCHS/SOL participants. It may be that residents who moved had foreclosure exposure inconsistent with the census tract associated with their health-related outcomes at the time of clinical measurement. While we are unable to determine how many participants this affects, approximately $14 \%$ of Hispanics/Latinos in the U.S. change residence in a given year, compared to $11 \%$ of non-Hispanic whites [33]. Healthier people with more resources to buffer the negative influence of adverse exposures in their neighborhood may selectively move to better neighborhoods with lower risk of foreclosure. As a result, the data showing better health outcomes in neighborhoods with a lower foreclosure risk could suffer from some selection bias. This is a common limitation of cross-sectional area-level studies that can best be addressed with a longitudinal study design. Another limitation of our study is that our data do not allow us to determine which residents are personally undergoing a foreclosure at the time of data collection. Prior research indicates that these residents may be more likely to experience stress due to their impending foreclosure status [34]. It is possible that the stress of an impending eviction may limit an individual's ability to access health promoting 
resources.. Our data only allow an examination of the relationship between living in a place with high foreclosure risk and CVD risk factors and is unable to determine the association of a personal foreclosure experienced by residents with CVD risk. Finally, as this study was conducted among Hispanic/Latino adults in four urban metropolitan areas, these results might not be generalizable to other populations. Additional research is needed to examine differences in foreclosure experiences and health affects among diverse racial and ethnic groups.

\section{Conclusion}

There are substantial burdens of cardiovascular disease risk factors in the U.S. among all major Hispanic/Latino background groups [35], and greater risks in low-income individuals [3]. As the pathways that contribute to these racial/ethnic and SES disparities in cardiovascular health are uncovered, housing has been identified as an important upstream social determinant that requires interdisciplinary interventions to address [36]. The growing evidence including the results from this study show that home mortgage foreclosures can negatively affect community health. Public health practitioners are increasingly looking outside of health care to housing policy to address many social and economic determinants of health [36, 37]. Housing policies that provide pathways to stable housing for homeowners and renters may bolster cardiovascular disease prevention campaigns within neighborhoods. For example, Making Home Affordable (MHA) programs are resources available to homeowners to help keep them in their homes should they be faced with the possibility of a foreclosure. Among renters, preventing eviction and displacement by implementing legislation that provides rental relief for tenants that pay more than $30 \%$ of their gross income on rent and utilities such as that proposed under the Rental Relief Act; or providing legal representation in housing court for low-income residents as is being done in New York City can be useful strategies to stabilize housing for vulnerable residents. Providing opportunities for residents to stay securely housed has the potential to curb the adverse health outcomes that accompany unstable housing.

\section{Abbreviations}

95\% Cl: 95\% Confidence Interval; APR: Adjusted Prevalence Ratio; BMI: Body Mass Index; CT: Census Tract; HCHS/SOL: Hispanic Community Health Study/ Study of Latinos; HUD: U.S. Department of Housing and Urban Development; PR: Prevalence Ratios

\section{Acknowledgements}

Not applicable.

\section{Funding}

This work was supported by the National Heart, Lung, and Blood Institute (NHLBI) grant K01HL125466 and 1R03HL140265 to Dr. Earle Chambers; and the Hispanic Community Health Study/Study of Latinos was carried out as a collaborative study supported by contracts from the National Heart, Lung, and Blood Institute (NHLBI) to the University of North Carolina (NO1-
HC65233), University of Miami (N01-HC65234), Albert Einstein College of Medicine (N01-HC65235), Northwestern University (N01-HC65236), and San Diego State University (N01-HC65237). The following Institutes/Centers/ Offices contribute to the HCHS/SOL through a transfer of funds to the NHLBI: National Center on Minority Health and Health Disparities, the National Institute of Deafness and Other Communications Disorders, the National Institute of Dental and Craniofacial Research, the National Institute of Diabetes and Digestive and Kidney Diseases, the National Institute of Neurological Disorders and Stroke, and the Office of Dietary Supplements. Dr. David Hanna was supported by K01HL137557.

\section{Availability of data and materials}

Data is available through the coordinating center of the HCHS/SOL (https:// sites.cscc.unc.edu/hchs/).

\section{Authors' contributions}

ECC, DBH, and SH contributed to the acquisition, analysis, and interpretation of the study data; and drafted the manuscript. DBH geocoded data and linked foreclosure data. DTD, MC, SNZ, JLM, KP, MDG, and LCG were major contributors to interpreting the housing and CVD data within the context of Hispanic health and writing and critically revising the manuscript. All authors read and approved the final manuscript prior to journal submission.

\section{Ethics approval and consent to participate}

The Institutional Review Boards of the Albert Einstein College of Medicine, the University of Illinois at Chicago, the University of Miami, San Diego State University, and the University of North Carolina at Chapel Hill (each field center and the coordinating center of the $\mathrm{HCHS} / \mathrm{SOL}$ ) approved the study protocol. All participants gave written informed consent in either English or Spanish.

\section{Consent for publication}

Not applicable.

Competing interests

The authors declare that they have no competing interests.

\section{Publisher's Note}

Springer Nature remains neutral with regard to jurisdictional claims in published maps and institutional affiliations.

\section{Author details}

${ }^{1}$ Department of Family \& Social Medicine, Albert Einstein College of Medicine, 1300 Morris Park Avenue, Harold and Muriel Block Building, Rm. 409, Bronx, NY 10461, USA. ${ }^{2}$ Department of Epidemiology \& Population Health, Albert Einstein College of Medicine, Bronx, NY 10461, USA. ${ }^{3}$ NYU Spatial Epidemiology Laboratory, Department of Population Health, New York University School of Medicine, New York, NY 10016, USA. ${ }^{4}$ Department of Community Health and Social Medicine, City University of New York School of Medicine, New York, NY 10031, USA. ${ }^{5}$ Department of Health Systems Science, University of Illinois at Chicago College of Nursing, Chicago, IL 60610, USA. 'Department of Medicine, Massachusetts General Hospital/ Harvard Medical School, Boston, MA 02114, USA. ${ }^{7}$ Department of Social Medicine, University of North Carolina, Chapel Hill, NC 27599-7240, USA.

${ }^{8}$ Behavioral Medicine Research Center, Department of Psychology, University of Miami, Miami, FL 33136, USA. 'Department of Psychology, San Diego State University, San Diego, CA 92120, USA.

Received: 18 April 2018 Accepted: 7 January 2019

Published online: 17 January 2019

\section{References}

1. Rugh JS, Massey DS. Racial segregation and the American foreclosure crisis. Am Sociol Rev. 2010;75(5):629-51.

2. Heart Disease Risk Factors [https://www.cdc.gov/heartdisease/risk_factors. htm].

3. Fryar CD, Chen T, Li X. Prevalence of uncontrolled risk factors for cardiovascular disease: United States, 1999-2010. NCHS data brief. 2012;103: $1-8$.

4. Arcaya M, Glymour MM, Chakrabarti P, Christakis NA, Kawachi I, Subramanian SV. Effects of proximate foreclosed properties on individuals' 
weight gain in Massachusetts, 1987-2008. Am J Public Health. 2013;103(9): e50-6.

5. Christine PJ, Moore K, Crawford ND, Barrientos-Gutierrez T, Sanchez BN, Seeman T, Diez Roux AV. Exposure to neighborhood foreclosures and changes in Cardiometabolic health: results from MESA. Am J Epidemiol. 2017;185(2):106-14.

6. Downing J, Karter A, Rodriguez H, Dow WH, Adler N, Schillinger D, Warton M, Laraia B. No spillover effect of the foreclosure crisis on weight change: the diabetes study of northern California (DISTANCE). PLoS One. 2016;11(3).

7. Christine PJ, Moore K, Crawford ND, Barrientos-Gutierrez T, Sanchez BN, Seeman T, Diez Roux AV. Exposure to neighborhood foreclosures and changes in Cardiometabolic health: results from MESA. Am J Epidemiol. 2016.

8. Arcaya M, Glymour MM, Chakrabarti P, Christakis NA, Kawachi I, Subramanian SV. Effects of proximate foreclosed properties on individuals' systolic blood pressure in Massachusetts, 1987 to 2008. Circulation. 2014; 129(22):2262-8.

9. Currie J, Tekin E. Is there a link between foreclosure and health? Am Econ JEcon Polic. 2015;7(1):63-94.

10. Tsai AC. Home Foreclosure, Health, and Mental health: a systematic review of individual, aggregate, and Contextual Associations. Plos One. 2015;10(4).

11. Pollack CE, Kurd SK, Livshits A, Weiner M, Lynch J. A case-control study of home foreclosure, health conditions, and health care utilization. J Urban Health. 2011;88(3):469-78.

12. Pollack CE, Lynch J. Health status of people undergoing foreclosure in the Philadelphia region. Am J Public Health. 2009;99(10):1833-9.

13. Alley DE, Lloyd J, Pagan JA, Pollack CE, Shardell M, Cannuscio C. Mortgage delinquency and changes in access to health resources and depressive symptoms in a nationally representative cohort of Americans older than 50 years. Am J Public Health. 2011;101(12):2293-8

14. Duncan D, Kawachi I. Neighborhoods and health. 2nd ed. Oxford, UK: Oxfodr University Press; 2018

15. Arcaya M. Neighborhood foreclosure and health. 2nd ed. Oxford, UK: Oxford University Press; 2018.

16. Lavange LM, Kalsbeek WD, Sorlie PD, Aviles-Santa LM, Kaplan RC, Barnhart J, Liu KA, Giachello A, Lee DJ, Ryan J, et al. Sample design and cohort selection in the Hispanic community health study/study of Latinos. Ann Epidemiol. 2010;20(8):642-9.

17. Sorlie PD, Aviles-Santa LM, Wassertheil-Smoller S, Kaplan RC, Daviglus ML, Giachello AL, Schneiderman N, Raij L, Talavera G, Allison M, et al. Design and implementation of the Hispanic community health study/study of Latinos. Ann Epidemiol. 2010;20(8):629-41.

18. HUD PROVIDED LOCAL LEVEL DATA [http://www.huduser.org/portal/ datasets/nsp_foreclosure_data.html].

19. Ortiz SE, Zimmerman FJ. Race/ethnicity and the relationship between homeownership and health. Am J Public Health. 2013;103(4):e122-9.

20. Schootman M, Deshpande AD, Pruitt SL, Jeffe DB. Neighborhood foreclosures and self-rated health among breast cancer survivors. Qual Life Res. 2012;21(1):133-41.

21. The 2007-2011 ACS 5-Year Summary File Technical Documentation. U.S. Departtment of Commence. Economic and Statistics Administration. U.S Census Bureau; 2012.

22. Duncan DT, Kawachi I, White K, Williams DR. The geography of recreational open space: influence of neighborhood racial composition and neighborhood poverty. J Urban Health. 2013;90(4):618-31.

23. Lindblad MR, Manturuk KR, Quercia RG. Sense of community and informal social control among lower income households: the role of homeownership and collective efficacy in reducing subjective neighborhood crime and disorder. Am J Community Psychol. 2013;51 (1-2):123-39.

24. Rossi PH, Weber E. The social benefits of homeownership: empirical evidence from National Surveys. Housing Policy Debate. 1996;7(1):1-35.

25. Rohe WM, Stewart LS. Homeownership and neighborhood stability. Housing Policy Debate. 1996;7(1):37-81.

26. Laaksonen M, Rahkonen O, Karvonen S, Lahelma E. Socioeconomic status and smoking. Eur J Pub Health. 2005;15(3):262-9.

27. Reid KW, Vittinghoff E, Kushel MB. Association between the level of housing instability, economic standing and health care access: a meta-regression. J Health Care Poor Underserved. 2008;19(4):1212-28.

28. Baggett TP, Lebrun-Harris LA, Rigotti NA. Homelessness, cigarette smoking and desire to quit: results from a US national study. Addiction. 2013;108(11): 2009-18.
29. Saegert S, Evans GW. Poverty, housing niches, and health in the United States. J Soc Issues. 2003;59(3):569-89.

30. Saegert S, Fields D, Libman K. Mortgage foreclosure and health disparities: serial displacement as asset extraction in African American populations. Journal of Urban Health-Bulletin of the New York Academy of Medicine. 2011;88(3):390-402.

31. Libman K, Fields D, Saegert S. Housing and health: a social ecological perspective on the US foreclosure crisis. Hous Theory Soc. 2012;29(1):1-24.

32. Roux AVD. The foreclosure crisis and cardiovascular disease. Circulation. 2014; 129(22):2248-9.

33. Ihrke D: Reason for moving: 2012-2013. In.: U.S. Department of Commerce Economics and Statistics Administration. U.S. CENSUS BUREAU 2014.

34. Cannuscio CC, Alley DE, Pagan JA, Soldo B, Krasny S, Shardell M, Asch DA, Lipman TH. Housing strain, mortgage foreclosure and health. Nurs Outlook. 2012:60(3):134-42.

35. Daviglus ML, Pirzada A, Talavera GA. Cardiovascular disease risk factors in the Hispanic/Latino population: lessons from the Hispanic community health study/study of Latinos (HCHS/SOL). Prog Cardiovasc Dis. 2014;57(3): 230-6.

36. Krieger J, Higgins DL. Housing and health: time again for public health action. Am J Public Health. 2002;92(5):758-68.

37. Libman $\mathrm{K}$, Fields $\mathrm{D}$, Saegert $\mathrm{S}$. Toward a research agenda at the intersections of housing and health. Hous Theory Soc. 2012;29(1):47-55.

\section{Ready to submit your research? Choose BMC and benefit from:}

- fast, convenient online submission

- thorough peer review by experienced researchers in your field

- rapid publication on acceptance

- support for research data, including large and complex data types

- gold Open Access which fosters wider collaboration and increased citations

- maximum visibility for your research: over $100 \mathrm{M}$ website views per year

At BMC, research is always in progress.

Learn more biomedcentral.com/submissions 